\title{
DEPARTMENTS
}

BO() K REVIEW S

\section{Fiber Optic Networks}

Paul E. Green, Jr., 539 pages, illus., index, references, list of symbols. ISBN 0-13-3194922. Prentice-Hall, Englewood Cliffs, NJ 07632 (1993) $\$ 59$ hardbound.

Reviewed by Dan B. Tolley, Hughes Information Technology Company, Hughes Communications and Data Systems, 1768 Business Center Drive, 4th Floor, Reston, VA 22090.

Until recently, fiber optics have generally been used as a point-to-point replacement for wire connections in the communications and computer industries. As applications and system requirements have progressed, researchers have begun to examine the potential use of fiber optics as a true interconnection mechanism, including optical switching and wavelengthdivision multiplexing (WDM) as key components for delivering data to users. Fiber Optic Networks is an excellent review of the components of interest and the current views of the possible local, metropolitan, and wide-area network topologies and structures that take advantage of fiber optic theory and practical devices.

Green has developed a book that can intrigue the reader with the potentially limitless opportunities presented by fiber optic interconnections, and he includes just enough pragmatism to allow the user to see that the transformation to optical networking will be a very challenging and imaginative process. The book gets to the heart of the problem by reminding the reader that computer and system designers may have to rethink the strategies of the past, based on the dramatic bandwidth capabilities $(20,000 \mathrm{Gbps})$ that are available and in response to device limitations (tuning, tuning speeds, power, coupling, crosstalk, etc.).

The book focuses on fiber optics as an integral part of the networks, not just a low-error, highspeed connection mechanism. The book is appropriate for a graduate-level curriculum or for providing the practicing engineer with a discus- sion of the challenges, problems, and limitations of fiber optic networking in today's world. The book relies heavily on references to provide detailed analyses of specialized topics, and limited outside reading may be required for users wishing a more complete understanding of the subtleties of fiber optics, fiber optic device physics, computer, and communications topics discussed. Green has selected the summations of the references well, allowing the book to flow freely and even generating a high level of interest in some of the references. My one complaint about the book would be lack of examples.

There are four sections to the book: Challenge, Building Blocks, Architecture, and Realization. The initial section, Challenge, defines the desire for networks that appear to us today as virtually error-free $\left(10^{-15}\right.$-bit error rate) with almost unlimited bandwidth (20,000 Gbps). Green is also quick to point out that even these bandwidths may appear severely limited in the future, as do today's 10-Mbps systems to us. The bandwidth limit in the future may occur because when transmitting at gigabit-per-second speeds, transmission setup times (even on the order of milliseconds) can allow the receiver to lose megabits of data. These rather significant setup times can also significantly affect the throughput of systems transferring kilobit files, since setup times can take 1000 times longer than the transmission (1000 bits can be transmitted in $10^{6} \mathrm{~s}$, while setup may take $10^{-3} \mathrm{~s}$ ). These types of issues call for faster setups or planned frequency or time-division utilization.

The Building Block section focuses on components involved in making an optical network and requires 325 pages. It includes WDM techniques requiring taps, fibers, filters, amplifiers, lasers, and many types of tunable devices. Each type of component is explained, equations and graphs are presented, and the impact on a system is also defined. Ample references are given if the reader wants to study the individual devices to a grater extent. This section could benefit from the additional examples discussed earlier, although
Green has presented the intuitive arguments involved in device performance, e.g., "In the future, if tunable filters of the three-port type can be made with low enough attenuation, they could be used in a bus geometry to avoid the energy wastage of the star geometry."

The Architecture section discusses topologies, network layering, network control, and protocol issues related to making fiber optic networks operate properly. Green has pointed to a need for common application profile interfaces (APIs), which are used by the applications to gain communications services. The use of APIs allows evolution of the communications components and software, without requiring rewrites of the application software. This section points to the need for lightweight protocols to minimize overhead requirements. The future network issues discussed include combinations of wave-, time-, and code-division multipleaccess capabilities and the related challenges of call setup, planning, and message selection. Included are quick discussions of circuits and packet switching requirements and how devices and system requirements can force solutions.

The final section, Realization, discusses the limited number of operational fiber optic networks available today, focusing mostly on successful telecommunications solutions. This section is extremely limited because most fiber optic networks that do exist are laboratory experiments and are not included.

Fiber Optic Networks is an extremely wellwritten book, presenting an overview of fiber optics as related to multi-user networks. The book contains the general equations of interest and ample references to satisfy any reader's interest. The tempo of the book is exciting and provides the user with a comprehensive understanding of the tasks required to make a fiber optic network a reality. The focus of the book is on providing the user with data in a responsive time frame and at a reasonable cost. Additional examples using the equations would be helpful, 
but the information is available to introduce the user to this new and exciting field of endeavor.

\section{Particle Field Holography}

Chandra S. Vikram, Vol. 11, Cambridge Studies in Modem Optics, Series Editors P. L. Knight and W. J. Firth, xviii + 265 pp., illus., bibliography, index. ISBN 0-521-41127-0. Cambridge University Press, The Pitt Building, Trumpington Street, Cambridge CB2 1RP, U.K. (1992) \$79.95 hardbound.

Reviewed by Peter Scott, State University of New York at Buffalo, Department of Electrical and Computing Engineering, Buffalo, NY 14260.

While the method called holography conjures visions of walk-around 3-D images and virtualreality computer displays, it continues to earn its daily bread by being good at important, if somewhat more pedestrian, tasks. One of the first practical applications of Dennis Gabor's invention, and still one of the most prominent, is the study of dynamic volumes of small particles. This is the subject of Chandra Vikram's monograph, a recent addition to the well-chosen list offered by Cambridge University Press in their Studies in Modern Optics series.

The volume contains 11 chapters, a bibliography with 440 entries, and a subject index. The chapters seem to divide into four clusters: history and basic science are introduced in Chaps. 1 through 3 , issues related to the technology of particle field holography are discussed in Chaps. 4 and 5 , reconstruction enhancement and its limits is the subject of Chaps. 6 through 9, and special techniques comprise the two concluding chapters.

Chapter 1 is a brief but useful historical introduction. The seminal contributions of Brian Thompson, who wrote the foreword, and those of Thompson's coworkers and students are properly emphasized.

Chapters 2 and 3 set out the basic notation and physical-mathematical models for scalar coherent image formation and reconstruction, with emphasis on the in-line far-field case. The purpose is limited to establishing an explicit framewoik for subsequent analysis of the particle field holographic application that is the subject of this book, and the conceptual development may be a bit thin for the uninitiated. Earlier, more broadly ranging monographs on holography by P. Hariharan [Optical Holography, Cambridge University Press, Cambridge, U.K. (1984)] and R. J. Collier, C.B. Burckhardt, and L. H. Lin [Optical Holography, Academic Press, New York (1971)] add scope and detail to this condensed presentation and might be kept on hand by those seeking a self-contained introduction. The mathematical level of this subject, incidentally, is not highly demanding, requiring only some familiarity with complex analysis and the wave equation.

Chapter 4 addresses system design considerations. Issues of finite spatiotemporal coherence, exposure requirements, and real image size and magnification are discussed. Of particular note is a careful analysis of the implications of particle motion in both lateral and transverse directions, an important problem to which Vikram has made notable research contributions.

Chapter 5, entitled "Practical Considerations," is by far the longest chapter and references over one quarter of all the citations. The chapter considers in depth various engineering aspects of image formation and reconstruction, including first-order errors in the idealized imaging model and strategies of compensation for them. Specific topics include relay lenses, aperture effects, practical recording media, exposure and reconstruction optical controls, transparent objects, and phase imaging. This chapter alone would justify the volume, pulling together as it does a considerable amount of recent literature on disparate but interrelated results that are of immediate relevance to the working holographer.

The point of the exercise is, of course, highresolution imaging of the cross-sectional shapes and 3-D spatial distribution of fine particles. Chapters 6 through 9 develop aspects of holographic reconstruction, including system design, reconstruction accuracy, and efficiency. Chapter 6 presents a formulation of holographic reconstruction at a level of detail commensurate with the forward-imaging model of Chaps. 2 and 3 , concentrating on the in-line far-field case. Chapter 7 discusses the subject of aberrations, the control of which is necessary to approach diffraction-limited resolution for the geometries of interest here. In Chap. 8, the question of fringe contrast is investigated. Although it is of general significance to the creation of bright holograms of all types, fringe contrast is particularly im-portant in the in-line case most commonly used for particle field work where the transmitted wave, empty of information or features, is expected to dominate energetically. Chapter 9 considers another question to which Vikram has made important original contributions (logether with coworker M. L. Billet), that is, analysis of the wave front in nonimage planes.

The book concludes with two brief chapters on problems at the present frontiers of coherent particle field imaging. Chapter 10 introduces issues involved in velocimetry and the holography of fast-moving particles, a focus of current research interest particularly for aerospace applications. A vision of cinematographic presentation of 3-D holographic data is also offered in this chapter. Finally, where particle density or large particle size precludes the wonderful simplicity of in-line holography (no optics required in the optical path), the off-axis technique pio- neered by E. N. Leith and J. Upatnieks for general 3-D imaging must be used. This is the subject of the final chapter.

The index, an important tool in a monograph of this kind, might have been expanded somewhat beyond its 300-odd entries. For instance, there were no entries for thick or thin holograms or for phase objects, subjects taken up at various places in this volume.

The appearance of a comprehensive monograph on particle field holography is long overdue and Vikram has drawn from a widely scattered literature to produce one notable for its conciseness, balance, and freedom from error. Of course, every reader with some interest in the field could quibble with the selection of topics and this reviewer is no exception. Additional discussion related to timely applications such as analysis of smokes and water and airborne pollutants and the recent trend toward computer-based reconstruction algorithms and systems would have been particularly welcome. Vikram's recent volume in the SPIE Milestone Series [Selected Papers in Holographic Particle Diagnostics, SPIE Milestone Series, Volume MS-21, Bellingham, WA (1990)] is helpful in this regard. Quibbles to the contrary notwithstanding, the present volume is most welcome and recommended for all with a scientific or engineering interest in particle field mensuration.

\section{BOOKS RECEIVED}

Phase Conjugate Optics by Jun-Ichi Sakai. 218 pp., illus., index, bibliography. ISBN 0-07054315-1. McGraw-Hill, Inc., 1221 Avenue of the Americas, New York, NY 10020 (1992) $\$ 52$ hardbound. Covers the history of phaseconjugate optics, basic concepts and properties of phase-conjugate light, theoretical foundations of the production of phase-conjugate light, generation and polarization of phase-conjugate light, wave equations with polarization, fourwave mixing in optical Kerr media, formulation of the coupled-mode equations, effects of absorption and pump depletion, special theories of four-wave mixing, qualitative explanation of resonant four-wave mixing, special mechanisms in four-wave mixing, transient response characteristics of four-wave mixing, principles of phase-conjugate light generation, experiments in phase-conjugate light generation, semiconductors, special mechanisms for four-wave mixing experiments, transient response characteristics of back degenerate four-wave mixing, phase-conjugate light generating media, sources of nonlinear polarization, dielectrics, semicondctors, band-filling effect, applications of phase-conjugate light, optical resonators, optical image transmission, semiconductor laser amplifiers, and optical pulse compression by stimulated Brillouin scattering. 